\title{
Quality of life drives patients' preferences for secondary findings from genomic sequencing
}

\author{
Chloe Mighton ${ }^{1,2} \cdot$ Lindsay Carlsson ${ }^{3,4} \cdot$ Marc Clausen $^{2} \cdot$ Selina Casalino $^{2} \cdot$ Salma Shickh $^{1,2} \cdot$ Laura McCuaig $^{2,5} \cdot$ \\ Esha Joshi ${ }^{2} \cdot$ Seema Panchal ${ }^{6} \cdot$ Kara Semotiuk $^{6} \cdot$ Karen Ott $^{7} \cdot$ Christine Elser $^{4,5,6}$. Andrea Eisen ${ }^{7}$. \\ Raymond H. Kim ${ }^{4,5,6}$. Jordan Lerner-Ellis ${ }^{6,8}$ - June C. Carroll ${ }^{6,9}$. Emily Glogowski ${ }^{10}$ - Kasmintan Schrader ${ }^{11,12}$. \\ Yvonne Bombard ${ }^{1,2}$ on behalf of the Incidental Genomics Study Team
}

Received: 28 November 2019 / Revised: 31 March 2020 / Accepted: 14 April 2020 / Published online: 18 May 2020

(c) The Author(s), under exclusive licence to European Society of Human Genetics 2020

\begin{abstract}
There is growing impetus to include measures of personal utility, the nonmedical value of information, in addition to clinical utility in health technology assessment (HTA) of genomic tests such as genomic sequencing (GS). However, personal utility and clinical utility are challenging to define and measure. This study aimed to explore what drives patients' preferences for hypothetically learning medically actionable and non-medically actionable secondary findings (SF), capturing clinical and personal utility; this may inform development of measures to evaluate patient outcomes following return of SF. Semistructured interviews were conducted with adults with a personal or family cancer history participating in a trial of a decision aid for selection of SF from genomic sequencing (GS) (www.GenomicsADvISER.com). Interviews were analyzed thematically using constant comparison. Preserving health-related and non-health-related quality of life was an overarching motivator for both learning and not learning SF. Some participants perceived that learning SF would help them "have a good quality of life" through informing actions to maintain physical health or leading to psychological benefits such as emotional preparation for disease. Other participants preferred not to learn SF because results "could ruin your quality of life," such as by causing negative psychological impacts. Measuring health-related and non-health-related quality of life may capture outcomes related to clinical and personal utility of GS and SF, which have previously been challenging to measure. Without appropriate measures, generating and synthesizing evidence to evaluate genomic technologies such as GS will continue to be a challenge, and will undervalue potential benefits of GS and SF.
\end{abstract}

Members of the Incidental Genomics Study Team are listed below acknowledgements.

Supplementary information The online version of this article (https:// doi.org/10.1038/s41431-020-0640-x) contains supplementary material, which is available to authorized users.

Yvonne Bombard

Yvonne.Bombard@utoronto.ca

1 Institute of Health Policy, Management and Evaluation, University of Toronto, Toronto, ON, Canada

2 Li Ka Shing Knowledge Institute of St. Michael's Hospital, Unity Health, Toronto, ON, Canada

3 Lawrence S. Bloomberg Faculty of Nursing, University of Toronto, Toronto, ON, Canada

4 University Health Network, Toronto, ON, Canada

5 Department of Medicine, University of Toronto, Toronto, ON, Canada

\section{Introduction}

Use of genomic sequencing (GS) is increasing both clinically and in research, and with it the generation of secondary findings (SF). We use the term SF to encompass a wide range of medically actionable and non-medically

6 Sinai Health System, Toronto, ON, Canada

7 Sunnybrook Health Sciences Centre, Toronto, ON, Canada

8 Department of Laboratory Medicine and Pathobiology, University of Toronto, Toronto, ON, Canada

9 Department of Family \& Community Medicine, University of Toronto, Toronto, ON, Canada

10 GeneDx, Gaithersburg, MD, USA

11 BC Cancer Agency, Vancouver, BC, Canada

12 Department of Medical Genetics, University of British Columbia, Vancouver, BC, Canada 
actionable results secondary to the primary reason for testing, beyond the American College of Medical Genetics and Genomics' recommended list of 59 medically actionable SF [1]. The types of SF that should be sought and disclosed have been widely debated. It is currently unknown whether SF will improve patients' outcomes through enabling early detection and prevention of disease, or if SF will lead to overdiagnosis, overtreatment, and excessive healthcare utilization [2]. There are currently gaps in the evidence on patient outcomes following genomic testing [3], and there is a need for health technology assessment (HTA) related to GS and SF.

HTA involves a synthesis of evidence on the validity, utility, economic, and health service impact of a new test or health technology, as well as evidence on ethical and social values at stake of adopting a test or technology [4, 5], with the primary goal to inform decision making about use of the technology in healthcare, be it at the patient/provider-, institutional- or regional/national/international level [6]. HTA is a fundamental determinant of reimbursement and implementation decisions related to new health technologies $[4,5]$. HTA for genetic and genomic technologies is challenged by a lack of overarching guidance on what outcomes should be measured, or what study designs confer appropriate evidence [7]. There is a need for greater consistency in approaches to generating and synthesizing evidence on genomic tests [7]. Further, availability and demand for genetic and genomic tests are increasing faster than systematic evaluation of such tests can be conducted [8]; there is a critical need for HTA to inform reimbursement decisions and evidence-based guidelines.

A key pillar of HTA is the evaluation of a test's clinical utility, the likelihood that a given test will improve health outcomes [5]. Clinical utility is typically measured through evaluating morbidity, mortality, clinical outcomes (e.g., a change in treatment), or health-related quality of life (QOL) associated with an intervention [5]. There are also calls to include personal utility, the nonclinical value of learning genetic information, in evaluations of genetic and genomic technologies [5, 8, 9]. Evaluating both the clinical and personal utility of genetic and genomic tests can be difficult [3]. Capturing the clinical utility of genetic and genomic tests is challenged by the fact that such tests ultimately provide only diagnostic or prognostic information [10], which may not be associated with measurable or immediate changes in treatment or a reduction in morbidity or mortality $[10,11]$. For instance, if a pathogenic variant in a gene associated with an autosomal dominant condition is identified in an asymptomatic individual (as can be the case with SF) there may be years or even decades between test delivery and disease onset [11]. There may also be multiple steps (e.g., active surveillance, medication, and surgery) between delivery of the test result and any associated health outcome (e.g., a reduction in mortality); all of these steps in the care pathway must be captured to fully measure the test's clinical utility [11]. Finally, SF may be associated with diseases that are not medically actionable. Providing patients with non-medically actionable SF may therefore not lead to any measurable change in clinical care, morbidity, or mortality.

Patients indicate value in learning both medically actionable and non-medically actionable results for reasons of personal utility [12-14]. Personal utility refers broadly to nonclinical outcomes of learning genetic test results and includes affective, cognitive, behavioral, and social domains $[5,15]$. Thus, evaluations of GS that focus solely on clinical endpoints will fail to capture the full scope of GS' impact. However, personal utility is inconsistently defined, and there are currently no standardized measures of the construct; nonclinical outcomes of GS are therefore difficult to evaluate [5, 15]. Evidence-based, standardized measures that capture nonclinical outcomes of GS are needed, and qualitative conceptual work is necessary to inform the development of such measures [16, 17].

In this study, we aimed to explore what drives patients' preferences for hypothetically learning five categories of medically actionable and non-medically actionable SF. A deeper understanding of patients' perceptions of clinical and personal utility related to a broad range of SF could inform the development of measures to capture outcomes following return of SF.

\section{Methods}

We used qualitative methodology informed by a grounded theory approach [18], and conducted semi-structured interviews to explore participants' hypothetical preferences for SF. We employed qualitative methods as they provide rich data on individual experiences and perspectives, appropriate for eliciting a deeper understanding of patients' preferences [19]. Ethics approval was obtained from the Research Ethics Boards at St. Michael's Hospital, Mount Sinai Hospital and Sunnybrook Health Sciences Center in Toronto, Canada.

\section{Design}

This was a qualitative study nested within a randomized control trial (RCT) of the effectiveness of a decision aid (www.GenomicsADvISER.com) for the selection of SF from GS. The RCT protocol and results are described elsewhere [20, 21]. All participants were patients with a personal cancer history or family history of a suspected hereditary cancer syndrome. Participants were recruited to the RCT from familial cancer genetics clinics in Toronto, 
Ontario, Canada, and had previously received an inconclusive test result from either single gene or panel testing. Participants in the RCT were randomized to either use the decision aid and speak with a genetic counselor over the phone (DA arm), or only speak with a genetic counselor via telephone (GC arm) to make their hypothetical selection of SF from GS. SF were categorized into five categories based on a proposed framework [22]: (1) Medically actionable results (e.g., those recommended by the American College of Medical Genetics and Genomics [1]), (2) Small increases in risk for common diseases (e.g., single nucleotide polymorphisms [SNPs] associated with type 2 diabetes), (3) Rare Mendelian diseases (e.g., DMD for muscular dystrophy), (4) Early-onset neurological diseases (e.g., PSEN1 for early-onset Alzheimer's disease, and (5) Carrier status (e.g., carrier of CFTR variant for cystic fibrosis).

\section{Participants and recruitment}

Participants were recruited from the sample of individuals $(n=133)$ enrolled in the RCT, from both arms of the trial. Sampling was initially purposive [23], and sought variation in SF category selection, clinical characteristics, and sociodemographic characteristics. After preliminary data analysis, theoretical sampling was employed [23] to maximize variation in participants' preferences for SF. For instance, we recruited participants whose category choices differed from those we had previously interviewed. Sampling continued until thematic saturation was reached [24], that is, no novel codes or themes were identified in the data [25].

\section{Data collection}

In depth, semi-structured telephone interviews were conducted between April and November 2017 by CM and LC. The interview guide (Appendix 1) was developed based upon the literature and study aims and modified based on emergent findings as data collection and analysis progressed. Interviews explored participants' SF category choices, with a specific focus on factors that informed category selection (e.g., actionability, uncertainty, and risk), their perceived utility of SF, and how they perceived utility and actionability across all SF categories. Data collection and data analysis were concurrent, which allowed for adaptation of the interview guide and sampling strategy based on themes that emerged from analysis. Interviews were audio recorded and transcribed verbatim. Interviewers took detailed field notes after each interview.

\section{Data analysis}

Transcripts were analysed using thematic analysis and constant comparison [18]. SC, LC, EJ, LM, and CM coded interviews, under the supervision of MC. The initial codebook was based upon the interview guide, research question, and data from the initial interviews. Regular team meetings were held to discuss participant recruitment, data collection, and analysis. Reflexivity was an element of these discussions; team members discussed how their own views and experiences may influence how they interpreted the data. Preliminary team meetings involved reviewing both interview audio files and transcripts, which led to the identification of new codes and themes from the data. The codebook was subsequently modified to incorporate novel codes and themes. To achieve consistency in coding, multiple coders coded 11 transcripts. Discrepancies between coders were resolved through discussion, and when necessary, the inclusion of a third coder. Data that arose under all topics in the interview guide were analyzed; themes related to perceptions of learning SF are the focus of this paper. Later analysis involved comparing contrasting cases where participants described conflicting perspectives on the utility and value associated with selecting SF. This enhanced the rigor of the analysis by identifying disconfirming evidence and resulted in a deeper interrogation of the previously identified themes. All analytic decisions and team meeting minutes were documented.

\section{Results}

\section{Participants' characteristics}

Thirty-one participants were interviewed. Interviews lasted on average $54 \mathrm{~min}$ (range $31-81 \mathrm{~min}$ ). Fifteen participants had used the decision aid and spoke with the study genetic counselor to select SF, and 16 had spoken with the study genetic counselor without using the decision aid. In our analysis, we did not find differences between those who used the decision aid and those who spoke with the genetic counselor. The majority of participants were female $(n=$ 28/31) (Table 1). About half were over 50 years old (16/31) and born in Canada (16/31). Most participants had completed post-secondary education $(n=19 / 31)$ and were working full-time (18/31). Most (20/31) were affected by cancer, primarily breast cancer (16/20). Most selected multiple SF categories (Table 2).

\section{Selection of SF categories was driven by how SF were perceived to affect quality of life}

Participants ultimately chose to learn or not learn categories of SF based on how they perceived these SF would affect different dimensions of their QOL. QOL was described broadly by participants, consisting of both health-related, and non-health-related dimensions. Typically, if participants 
Table 1 Participants' sociodemographic and clinical characteristics. $(n=31)$.

\begin{tabular}{|c|c|c|}
\hline \multirow[t]{2}{*}{ Sex } & Female & $28(90 \%)$ \\
\hline & Male & $3(9 \%)$ \\
\hline \multirow[t]{2}{*}{ Age } & $18-49$ & $15(48 \%)$ \\
\hline & 50 and over & $16(51 \%)$ \\
\hline \multirow[t]{7}{*}{ Race/ethnicity } & Black, Caribbean & $1(3 \%)$ \\
\hline & East Asian & $2(9 \%)$ \\
\hline & Latin American & $1(3 \%)$ \\
\hline & Mixed heritage & $4(12 \%)$ \\
\hline & Southeast Asian & $1(3 \%)$ \\
\hline & White/European & $20(64 \%)$ \\
\hline & Other & $2(6 \%)$ \\
\hline \multirow[t]{2}{*}{ Education } & College, High school, or less & $12(38 \%)$ \\
\hline & $\begin{array}{l}\text { Bachelor degree or } \\
\text { postgraduate degree }\end{array}$ & $19(61 \%)$ \\
\hline \multirow[t]{2}{*}{ Income } & $\$ 79,000$ or less & $13(41 \%)$ \\
\hline & $\$ 80,000$ or more & $15(48 \%)$ \\
\hline \multirow[t]{2}{*}{ Country of Origin } & Outside Canada & $15(48 \%)$ \\
\hline & Canada & $16(51 \%)$ \\
\hline $\begin{array}{l}\text { Family history } \\
\text { of cancer }\end{array}$ & Yes & $31(100 \%)$ \\
\hline \multirow[t]{3}{*}{ Affected by cancer } & Yes & $20(64 \%)$ \\
\hline & No & $10(32 \%)$ \\
\hline & Unsure & $1(3 \%)$ \\
\hline \multirow[t]{6}{*}{ Cancer type $^{\mathrm{a}}$} & Breast & $16(51 \%)$ \\
\hline & Endometrial & $2(6 \%)$ \\
\hline & Ovarian & $2(6 \%)$ \\
\hline & Colon & $1(3 \%)$ \\
\hline & Thyroid & $1(3 \%)$ \\
\hline & $B R C A 1$ and/or BRCA2 only & $16(51 \%)$ \\
\hline \multirow[t]{4}{*}{$\begin{array}{l}\text { Participant-reported } \\
\text { past genetic testing }\end{array}$} & $\begin{array}{l}\text { Hereditary breast and ovarian } \\
\text { cancer genes, other than } B R C A 1 / 2\end{array}$ & $3(9 \%)$ \\
\hline & $\begin{array}{l}B R C A 1 / 2 \text { and other hereditary } \\
\text { breast and ovarian cancer genes }\end{array}$ & $4(12 \%)$ \\
\hline & Lynch syndrome & $3(9 \%)$ \\
\hline & Unsure & $5(16 \%)$ \\
\hline
\end{tabular}

${ }^{a}$ Two patients were affected by multiple cancers (thyroid and endometrial, ovarian, and breast).

perceived that learning a category would have an overall positive impact on their QOL, then they would select to learn that category of SF. Many participants described a sense of expanded actionability, perceiving all types of SF as "actionable," either through medical, lifestyle, or practical actions such as planning:

"Everything that I've seen as examples here, there is action that can be taken, either in my own life or the life of someone that is close to me through this knowledge." -Pt 09 (GC arm)
This sense of expanded actionability was linked to the idea that learning SF across all categories could inform actions that could contribute to QOL:

"I've got to know everything. And, if it can't be treated well, so be it, but what other steps can I take to have a good quality of life?" -Pt 19 (GC arm)

Despite understanding limitations of SF (e.g., uncertain predictive value), which were explored in interviews, a number of participants expressed that the perceived benefits to their QOL outweighed the limitations and chose to learn all categories. A perceived benefit cited by many participants was the inherent value of having more information about their health, for instance, that "the more information people have, the better" (Pt 02, GC arm). Information was described as valuable for its own sake, as well as to be used to improve overall QOL:

"I think more information is better than less. Even if it isn't for the goal at hand, I think it's important for someone to be able to control their destiny and their future well-being." -Pt 06 (DA arm)

Conversely, other participants described how learning specific categories of SF would in fact be detrimental to their QOL, for instance by leading to negative psychological impacts or making them overly vigilant about disease onset. This was particularly salient for categories in which participants perceived that they had no control over disease course or outcome. If learning results from a particular category of SF was perceived to overall be detrimental to their QOL, participants chose not to learn that category:

"I think I just wouldn't want to know. [...] It would be driving me crazy trying to figure out, am I going to get it or not? And, it could ruin your quality of life." - Pt 25 (DA arm)

Participants described impacts of SF on several dimensions of their QOL: physical health, emotional and psychological well-being, and practical preparations for disease.

\section{Dimension of QOL: physical health}

Participants described how learning SF across all categories could improve their physical health through informing actions to prevent disease, delay disease onset or manage symptoms, thereby improving QOL. For instance:

"I would take anything [any SF]. Yeah. Absolutely. If you don't know something's broke, you can't fix it. 
Table 2 Participants' choices of secondary findings.

\begin{tabular}{lccccc}
\hline $\begin{array}{c}\text { Category 1: } \\
\text { Medically } \\
\text { actionable } \\
\text { diseases }\end{array}$ & $\begin{array}{c}\text { Category 2: } \\
\text { Common } \\
\text { disease SNPs }\end{array}$ & $\begin{array}{c}\text { Category 3: } \\
\text { Rare Mendelian } \\
\text { disorders }\end{array}$ & $\begin{array}{c}\text { Category 4: } \\
\text { Early-onset } \\
\text { neurological } \\
\text { conditions }\end{array}$ & $\begin{array}{c}\text { Category 5: } \\
\text { Carrier status }\end{array}$ \\
\hline Yes & $30(96.8 \%)$ & $24(77.4 \%)$ & $21(67.7 \%)$ & $20(64.5 \%)$ & $23(74.2 \%)$ \\
No & $1(3.2 \%)$ & $6(19.4 \%)$ & $6(19.4 \%)$ & $7(22.6 \%)$ & $5(16.1 \%)$ \\
Unsure & $0(0 \%)$ & $1(3.2 \%)$ & $4(12.9 \%)$ & $4(12.9 \%)$ & $3(9.7 \%)$ \\
\hline
\end{tabular}

That's the difference. That's my attitude. If you tell me it's broken and you tell me what I got to do to help lengthen my life or quality of life, I'll do it." -Pt 02 (GC arm)

For results in Category 1: medically actionable diseases, participants described the importance of taking "action" to prevent disease and subsequently preserve their physical health. Some participants prioritized medical interventions overall other types of actions that could be informed by learning SF. In fact, most described Category 1: medically actionable diseases as being the most valuable type of SF to learn. Indeed, all participants but one chose to learn Category 1: medically actionable diseases.

Even when considering SF that were not classified as medically actionable (i.e., categories other than Category 1), many participants described actions they could take to improve or preserve their physical health overall, which may delay or prevent disease onset. For instance, participants discussed reducing disease risk or delaying disease onset through changing their diet, exercise habits and other lifestyle factors. Some participants described that SF could motivate them to adopt better health habits for which they currently lacked motivation. While participants understood that there were no established medical preventions or treatments, they described actions such as "brain games" to reduce risk for or delay Alzheimer's disease, and one participant described her willingness towards "taking anything that doesn't have a harm" (Pt 07, DA arm) to reduce her risk for early-onset neurological conditions. Others reflected on how diseases that are not medically actionable now may be medically actionable in the future, as scientific and medical knowledge improve. Participants also discussed how the perceived benefits to QOL extended beyond themselves to family members, and described the value of sharing SF to improve family members' health and QOL:

"[I would share my results with] everybody. Well, I mean everybody who wanted to know. [...] Just keep it alive in the family, so that it's something that we can all use to...you know, to improve everybody's health and quality of life." -Pt 18 (DA arm)

Conversely, some participants reflected on how learning SF, specifically results associated with diseases without preventive options or treatment, may make them overly vigilant about disease symptoms, and lead to unnecessary physician visits. This was described as something that would detract from QOL:

"To me, the wondering would kill me. And I think it would ruin every aspect of my life, because it would totally consume me. [...] And then I'm going to spend more time thinking about that when I should be in there talking to my kids. Or sitting in a doctor's office waiting for results of a...you know, completely fabricated...what's the word? Symptom, right that I completely made up, instead of doing other things, so no. I definitely don't want to." - Pt 18 (DA Arm)

\section{Dimension of QOL: emotional and psychological well-being}

Some participants described how learning SF could positively contribute to their emotional and psychological wellbeing, both immediately and in the event of a future disease, to the benefit of their QOL. For instance, participants described their desire to learn as much information as possible about their health, and how learning more information about their health, i.e., SF, was inherently of value to them, and could lead to emotional benefits such as a sense of control. Participants also described that learning SF could allow them to emotionally prepare for the possibility of developing diseases, rather than being surprised if the disease occurred. Some participants described how learning SF may lead them to change their outlook on life, encouraging them to, "liv[e] your own life in the moment as opposed to putting it off until next week" (Pt 10, GC arm).

Conversely, other participants described how learning SF could detract from their emotional and psychological wellbeing. This was particularly salient for categories where participants did not perceive they had control over disease course or outcome, such as Category 3: rare Mendelian diseases and Category 4: early-onset neurological diseases, where they perceived the diseases as being severe or "devastating" (Pt 09, GC arm). In addition, the one participant who chose not to learn results from Category 1: medically actionable diseases did not choose that category 
because the potential for anxiety about disease risk outweighed the potential benefits of disease prevention. Other participants also described how they might constantly ruminate about whether or when they would develop diseases indicated by SF, to the detriment of their QOL:

\section{"I probably thought that whatever my actions aren't going to make a difference to this disease state coming upon me, then I don't really need to know it. Because then I may be stressed out anticipating it all the time, right, so I would just try to live my life happily and without this fear hanging over me." - Pt 01 (DA arm)}

Some participants reflected on the potential for SF to have negative psychological impacts for themselves and their family members and described feeling conflicted about sharing results with family members. Others described the possibility for guilt about potentially passing harmful conditions onto their children.

\section{Dimension of QOL: practical preparations for disease}

Participants discussed how SF could inform actions related to planning or preparing for the future, to maintain QOL in the event of an illness. This was salient for all categories of $\mathrm{SF}$, even those where diseases are not treatable or preventable. For instance, participants described how learning SF could inform their financial planning, allowing them to get their finances in order prior to disease onset. Some participants described how learning SF could motivate them to ensure that their family and social networks would support them in the event of future illness. Others discussed how SF could motivate them to travel more frequently earlier in life, to allow them to see everything they wanted to see prior to becoming ill. Participants also discussed practical preparations such as arranging accessible housing if they were at risk to develop illnesses that could affect their mobility. One participant described how, if she learned she were at risk for a condition that would cause blindness, she would learn Braille in advance. All of these actions were perceived as preserving participants' QOL.

\footnotetext{
"Say if I were to be going blind, I would be learning Braille now, so that I would be very fluent in it and not going to be stuck having to have someone read me all of these things. Or, if there is certain software that you need for your computer, for voice-overs and stuff like that, getting that in place now. Or, if, say I was to go blind when I'm 65, getting those in place when I'm 40, or something like that. Or, if I were to develop muscular dystrophy, move into a house that is more
}

accessible. [...] [For me, in the context of Category 3: rare Mendelian diseases, actionability means] the preparedness for when this [disease] does happen. You can't prevent the disease in itself, but you can take actions to make your life easier when it does happen, or as it starts to develop." -Pt 24 (GC arm)

Participants also described potential practical benefits to relatives. For instance, they described how they could alleviate burden from their children by putting their finances in order in advance if they knew they were at risk for a serious neurodegenerative illness. They also described potential benefits of informing relatives of carrier status results to inform their reproductive decision making. These actions could potentially improve QOL for relatives.

\section{Personal utility, clinical utility, and quality of life were related}

Participants described the value of SF in ways that aligned with personal utility and clinical utility. For participants, personal utility had two components: the actions that could be taken on the basis of SF (e.g., planning, sharing with relatives), and the inherent value of the information ("knowledge is power" [Pt 16, GC arm]). Moreover, personal utility seemed to overlap with clinical utility, suggesting that these concepts may lay on a continuum. This proposed relationship between personal utility, clinical utility and quality of life is illustrated in Fig. 1. As depicted in the continuum, some actions described by participants, such as planning, or changing outlook on life, were consistent with personal utility. Others, such as disease prevention through clinical actions, were aligned with the traditional definition of clinical utility. However, participants also described other actions or utilities that fell in between the personal/clinical utility divide, including patient-initiated lifestyle changes that aimed to reduce disease risk or delay onset (e.g., exercise, diet changes, brain games), or psychological outcomes such as anxiety or distress. QOL encompassed all of the dimensions of personal and clinical utility, and was the overarching outcome that participants would hope to achieve through learning SF.

\section{Discussion}

This study identified QOL as an overarching motivator in participants' decisions to both learn and not learn SF from GS. Given the challenges of measuring clinical and personal utility, QOL may serve as a comprehensive endpoint of personal and clinical utility since it captures how participants perceive the health and non-health-related impacts of SF. These findings are timely, given the 
Fig. 1 Proposed relationship between personal utility, clinical utility, and quality of life. Personal utility overlapped with clinical utility, suggesting that these concepts lie on a continuum. Examples of actions described by participants are placed along the continuum. QOL encompassed all of the dimensions of personal and clinical utility.

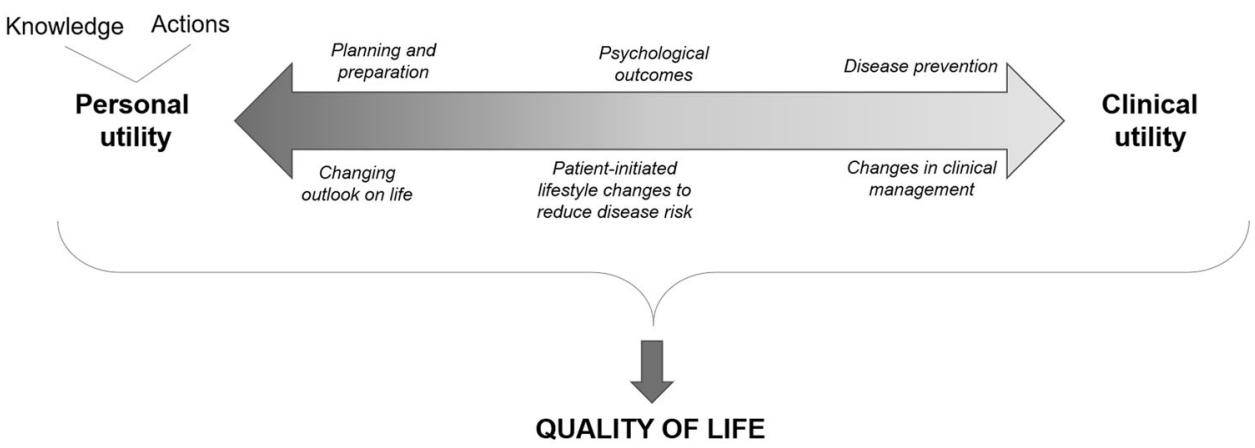

accelerated use of GS in clinical settings and the need for evaluation of the health and non-health outcomes of delivering SF to patients.

Our results extend others' findings on QOL related to genetic and genomic testing, which have primarily focused on health-related QOL. Prior studies have found that individuals who received genetic tests with established clinical utility reported higher improvements to QOL than those who had tests associated with less established clinical utility [26]. Our findings suggest that patients anticipate that SF without established medical actions may also contribute to QOL. Others report that extending QOL was a motivator for individuals to learn about variants that affect treatment response [27], and that QOL motivated participants to choose to learn medically actionable results [28]. We found that QOL was a motivator for wanting to learn both medically actionable and non-medically actionable results. Interestingly, QOL was also found to be a motivator to not want to learn SF. This suggests that QOL is a relevant outcome for individuals who learn SF and those who do not learn SF, which could enable comparisons between those groups in evaluation of GS.

Our participants' descriptions of the value of SF align with elements of personal utility found throughout the genetic testing literature. These include the utility of SF to enable mental preparation and future planning, the ability to share results with family members, and the inherent value of information [15, 29, 30]. Evaluations of genetic and genomic tests in cancer screening increasingly consider dimensions of personal utility in addition to traditional metrics of morbidity and mortality, which constitutes a departure from traditional screening paradigms [8]. Similarly, as GS is currently being implemented more broadly in clinical care, evaluation of GS across all indications should include domains of personal utility to capture the full scope of the test's outcomes. However, there are currently no standardized measures of personal utility, which is a barrier to the inclusion of personal utility in HTA; QOL could serve to bridge this gap. It may be possible to apply or adapt existing QOL measures to capture the non-health-related benefits and harms of SF.
In contexts outside of genetics, scales that capture broad domains of health, and non-health-related QOL have been developed. For instance, the World Health Organization (WHO) identifies physical, psychological, social, and environmental domains to QOL, which can be captured with the WHOQOL-BREF QOL assessment tool [31]. These domains are consistent with our participants' descriptions of how SF may affect their QOL. Interestingly, the WHOQOL-BREF includes an item that addresses access to information, which aligns with the value that participants in this study placed on accessing information about their health [31]. The Ferrans and Powers Quality of Life (QLI) instrument also measures broad domains of QOL that converge with our findings: health and functioning, socioeconomic, psychological/spiritual, and family [32]. Items on the scale assess health-related QOL for the individual completing the survey in addition to how they perceive their family members' health, as well as their own non-healthrelated QOL such as feelings of control and emotional support, all of which align with constructs discussed by our participants. Applying existing QOL measures or adapting them for use in the context of GS and SF may be useful to evaluate patients' self-reported QOL before and after receiving $\mathrm{SF}$ and GS. To our knowledge, these measures have not yet been applied in this context. There is a need for further research to determine how existing measures of QOL perform in the context of genetic and genomic testing, and consensus on which measures are most appropriate for use in this context.

HTA traditionally employs measures of health-related QOL to calculate quality-adjusted life years for cost-utility analysis in hypothetical health states as well as lived health states [33-35]; one example is the EQ-5D that captures mobility, self-care, usual activities, pain/discomfort, and anxiety/depression [34]. However, health-related QOL measures may not be sensitive enough to capture all benefits and harms of genetic testing [34] and may miss dimensions of QOL described as being important to participants (e.g., value of information). QOL measures that capture both health-related and non-health-related QOL may be able to assess genetic tests more comprehensively, though these 
measures are not traditionally used in HTA. Indeed, scholars have recently argued that there is a need for more patientcentered evaluations of genomic tests, through the incorporation of outcomes that matter to patients; others have called for the evaluation of genomic tests to involve more patient-centered definitions of utility [3, 36]. Given our participants' emphasis on the importance of health- and nonhealth-related QOL, evaluating non-health-related dimensions of QOL in addition to health-related QOL could contribute to more patient-centered evaluations of GS.

Our study has several limitations. Patients made a hypothetical decision about learning SF; perceptions of the utility of SF may differ for actual decisions. However, given that this study focused on exploration of patients' perceptions, toward developing patient-centered outcome measures, this limitation may not be relevant. In addition, all patients in our sample had a personal and/or family history of cancer, which may limit the transferability of our findings to non-cancer contexts. However, GS is increasingly offered in this population, making an exploration of these patients' views relevant. In addition, all participants had previously received single gene or panel testing, which suggests that they are amenable to genetic testing and their views may not reflect the views of individuals who decline genetic testing. Further research could explore how previous genetic testing experience may shape views and expectations for GS. Finally, our participants were predominantly female, educated, affected by cancer, and of European descent. These characteristics may limit transferability of our findings to individuals whose characteristics do not match our study participants. More research is needed with larger and more diverse populations.

\section{Conclusion}

This study identified QOL as an overarching motivator in participants' decisions about learning SF, which supports the use of QOL as an endpoint in the evaluation of genetic and genomic tests to capture patient outcomes that have previously been challenging to measure. This may be accomplished with existing measures that broadly capture QOL, such as the WHOQOL-BREF [31] or the QLI [32], or by developing or adapting measures for use in the context of GS and SF. Measuring health-related and non-health-related QOL may capture outcomes related to clinical and personal utility of GS and SF. Without comprehensive, sensitive and consistent measures, generating and synthesizing evidence to evaluate genomic technologies such as GS will continue to be a challenge; evaluations may undervalue potential benefits of GS and SF, or may mis-assess potential benefits and harms.
Acknowledgements We would like to thank the following individuals for supporting this study: Carolyn Piccinin, Laura Winter-Paquette, Melyssa Aronson, Talia Mancuso, Justin Lorentz, Tracy Graham, Yael Silberman, Rochelle Demsky, Alexandra Volenik, Jeanna McCuaig, Oana Morar, Leslie Ordal, and Nicholas Watkins. We would like to thank Theresa H.M. Kim for her contributions to the statistical analysis and recruitment in the RCT. This research was supported by grants from the Canadian Institutes of Health Research (CIHR) and the University of Toronto McLaughlin Center awarded to YB (\#333703 and MC-2016-04 respectively). YB was supported by a CIHR New Investigator Award during this study. CM received support from the Research Training Center at St. Michael's Hospital, the Canadian Institutes of Health Research (\#160968, GSD-164222) and a studentship funded by the Canadian Center for Applied Research in Cancer Control (ARCC). ARCC receives core funding from the Canadian Cancer Society (Grant \#2015-703549). Finally, we would like to thank our interview participants for their time and valuable insights.

Incidental Genomics Study Team Yvonne Bombard (PI), ${ }^{1,2}$ Susan Randall Armel, ${ }^{4,13}$ Melyssa Aronson, ${ }^{6,13}$ Nancy Baxter, ${ }^{1,2,14}$ Ken Bond, ${ }^{15}$ José-Mario Capo-Chichi, ${ }^{4,8}$ June C. Carroll, ${ }^{6,9}$ Timothy Caulfield, ${ }^{16,17,18}$ Marc Clausen, ${ }^{2}$ Tammy J. Clifford, ${ }^{19}$ Iris Cohn, ${ }^{20}$ Irfan Dhalla, ${ }^{1,2,5,21,22}$ Craig C. Earle, ${ }^{7,22,23}$ Andrea Eisen, ${ }^{7}$ Christine Elser, ${ }^{4,5,6}$ Mike Evans, ${ }^{2}$ Emily Glogowski, ${ }^{10}$ Tracy Graham, ${ }^{7}$ Jada G. Hamilton, ${ }^{24}$ Wanrudee Isaranuwatchai, ${ }^{1,2}$ Monika Kastner, ${ }^{1,2,9}$ Raymond H. Kim, ${ }^{4,5,6}$ Andreas Laupacis, ${ }^{1,2}$ Jordan Lerner-Ellis, ${ }^{6,8}$ Chantal F. Morel, ${ }^{4}$ Michelle Mujoomdar, ${ }^{25}$ Kenneth Offit, ${ }^{24}$ Seema Panchal, ${ }^{6}$ Mark Robson, ${ }^{24}$ Stephen W. Scherer, ${ }^{5,13,20}$ Adena Scheer, ${ }^{2,14}$ Kasmintan Schrader, ${ }^{11,12}$ Terrence Sullivan ${ }^{1}$ and Kevin E. Thorpe ${ }^{26,27}$

${ }^{13}$ Department of Molecular Genetics, University of Toronto,
Toronto, ON, Canada; ${ }^{14}$ Department of Surgery, University of Tor-
onto, Toronto, ON, Canada; ${ }^{15}$ Institute of Health Economics,
Edmonton, AB, Canada; ${ }^{16} \mathrm{Faculty}$ of Law, University of Alberta,
Edmonton, AB, Canada; ${ }^{17} \mathrm{School}$ of Public Health, University of
Alberta, Edmonton, AB, Canada; ${ }^{18} \mathrm{Health}$ Law Institute, University of
Alberta, Edmonton, AB, Canada; ${ }^{19} \mathrm{School}$ of Epidemiology and
Public Health, University of Ottawa, Ottawa, ON, Canada; ${ }^{20} \mathrm{The}$
Hospital for Sick Children, Toronto, ON, Canada; ${ }^{21}$ Health Quality
Ontario, Toronto, ON, Canada; ${ }^{22}$ Institute for Clinical Evaluative
Sciences, Toronto, ON, Canada; ${ }^{23}$ Canadian Partnership Against
Cancer, Toronto, ON, Canada; ${ }^{24}$ Memorial Sloan Kettering Cancer
Center, New York, NY, USA; ${ }^{25}$ Canadian Agency for Drugs and
Technologies in Health, Ottawa, ON, Canada; ${ }^{26}$ Applied Health
Research Centre (AHRC), Li Ka Shing Knowledge Institute of St.
Michael's Hospital, Unity Health, Toronto, ON, Canada; ${ }^{27}$ Dalla Lana
School of Public Health, University of Toronto, Toronto, ON, Canada

Funding YB received funding for this study from Canadian Institutes of Health Research (CIHR, \#333703), the University of Toronto McLaughlin Center (MC-2016-04) and a CIHR New Investigator Award. CM received support from the Research Training Center at St. Michael's Hospital, CIHR (FRN \#160968, FRN GSD-164222) and a studentship from the Canadian Center for Applied Research in Cancer Control (ARCC) which receives core funding from the Canadian Cancer Society (Grant \#2015-703549).

\section{Compliance with ethical standards}

Conflict of interest The authors declare that they have no conflict of interest. 
Publisher's note Springer Nature remains neutral with regard to jurisdictional claims in published maps and institutional affiliations.

\section{References}

1. Kalia SS, Adelman K, Bale SJ, Chung WK, Eng C, Evans JP, et al. Recommendations for reporting of secondary findings in clinical exome and genome sequencing, 2016 update (ACMG SF v2.0): a policy statement of the American College of Medical Genetics and Genomics. Genet Med. 2017;19:249-55.

2. Wilson BJ, Miller FA, Rousseau F. Controversy and debate on clinical genomics sequencing-paper 1: genomics is not exceptional: rigorous evaluations are necessary for clinical applications of genomic sequencing. J Clin Epidemiol. 2017;92:4-6.

3. Phillips KA, Deverka PA, Sox HC, Khoury MJ, Sandy LG, Ginsburg GS, et al. Making genomic medicine evidence-based and patient-centered: a structured review and landscape analysis of comparative effectiveness research. Genet Med. 2017;19: 1081-91.

4. Ungar W. Next generation sequencing and health technology assessment in autism spectrum disorder. J Can Acad Child Adolesc Psychiatry. 2015;24:123-7.

5. Bombard Y, Bach PB, Offit K. Translating genomics in cancer care. J Natl Compr Cancer Netw. 2013;11:1343-53.

6. Goodman CS. HTA 101: introduction to health technology assessment. Bethesda, MD: National Library of Medicine; 2014.

7. Garfield S, Polisena J, S Spinner D, Postulka A, Y Lu C, Tiwana SK, et al. Health technology assessment for molecular diagnostics: practices, challenges, and recommendations from the medical devices and diagnostics special interest group. Value Health. 2016;19:577-87.

8. Hamilton JG, Edwards HM, Khoury MJ, Taplin SH. Cancer screening and genetics: a tale of two paradigms. Cancer Epidemiol Biomark Prev. 2014;23:909-16.

9. Pitini E, De Vito C, Marzuillo C, D'Andrea E, Rosso A, Federici A, et al. How is genetic testing evaluated? A systematic review of the literature. Eur J Hum Genet. 2018;26:605-15.

10. Botkin JR, Teutsch SM, Kaye CI, Hayes M, Haddow JE, Bradley LA, et al. Outcomes of interest in evidence-based evaluations of genetic tests. Genet Med. 2010;12:228-35.

11. Veenstra DL, Piper M, Haddow JE, Pauker SG, Klein R, Richards $\mathrm{CS}$, et al. Improving the efficiency and relevance of evidencebased recommendations in the era of whole-genome sequencing: an EGAPP methods update. Genet Med. 2013;15:14-24.

12. Mighton C, Carlsson L, Clausen M, Casalino S, Shickh S, McCuaig L, et al. Development of patient "profiles" to tailor counseling for incidental genomic sequencing results. Eur J Hum Genet. 2019;27:1008-17.

13. Kohler JN, Turbitt E, Lewis KL, Wilfond BS, Jamal L, Peay HL, et al. Defining personal utility in genomics: a Delphi study. Clin Genet. 2017;92:290-7.

14. Lupo PJ, Robinson JO, Diamond PM, Jamal L, Danysh HE, Blumenthal-Barby J, et al. Patients' perceived utility of wholegenome sequencing for their healthcare: findings from the MedSeq project. Per Med. 2016;13:13-20.

15. Kohler JN, Turbitt E, Biesecker BB. Personal utility in genomic testing: a systematic literature review. Eur J Hum Genet. 2017;25:662-8.

16. Morse J, Mitcham C, Hupcey J, Tason M. Criteria for concept evaluation. J Adv Nurs. 1996;24:385-90.

17. Morse J, Field P. Qualitative research methods for health professionals. Thousand Oaks, CA: Sage Publications; 1995.
18. Strauss AL, Corbin J. Basics of qualitative research: grounded theory procedures and techniques. Newbury Park, CA: Sage; 1990.

19. Charmaz KC. Constructing grounded theory: a practical guide through qualitative analysis. London: SAGE Publications Ltd.; 2006.

20. Shickh S, Clausen M, Mighton C, Casalino S, Joshi E, Glogowski $\mathrm{E}$, et al. Evaluation of a decision aid for incidental genomic results, the Genomics ADvISER: protocol for a mixed methods randomised controlled trial. BMJ Open. 2018;8:e021876.

21. Bombard Y, Clausen M, Shickh S, Mighton C, Casalino S, Kim THM, et al. Effectiveness of the Genomics ADvISER decision aid for the selection of secondary findings from genomic sequencing: a randomized clinical trial. Genet Med. 2020;22:727-35.

22. Berg JS, Khoury MJ, Evans JP. Deploying whole genome sequencing in clinical practice and public health: meeting the challenge one bin at a time. Genet Med. 2011;13:499-504.

23. Coyne IT. Sampling in qualitative research. Purposeful and theoretical sampling; merging or clear boundaries? J Adv Nurs. 1997;26:623-30.

24. Morse J. The significance of saturation. Qual Health Res. 1995;5:147-9.

25. Saunders B, Sim J, Kingstone T, Baker S, Waterfield J, Bartlam B, et al. Saturation in qualitative research: exploring its conceptualization and operationalization. Qual Quant. 2018;52:1893-907.

26. D'Andrea E, Lagerberg T, De Vito C, Pitini E, Marzuillo C, Massimi A, et al. Patient experience and utility of genetic information: a cross-sectional study among patients tested for cancer susceptibility and thrombophilia. Eur J Hum Genet. 2018;26:518-26.

27. Kaphingst KA, Ivanovich J, Biesecker BB, Dresser R, Seo J, Dressler LG, et al. Preferences for return of incidental findings from genome sequencing among women diagnosed with breast cancer at a young age. Clin Genet. 2016;89:378-84.

28. Wright MF, Lewis KL, Fisher TC, Hooker GW, Emanuel TE, Biesecker LG, et al. Preferences for results delivery from exome sequencing/genome sequencing. Genet Med. 2014;16:442-7.

29. Hamilton JG, Shuk E, Genoff MC, Rodriguez VM, Hay JL, Offit $\mathrm{K}$, et al. Interest and attitudes of patients with advanced cancer with regard to secondary germline findings from tumor genomic profiling. J Oncol Pract. 2017;13:e590-601.

30. Turrini M, Prainsack B. Beyond clinical utility: the multiple values of DTC genetics. Appl Transl Genom. 2016;8:4-8.

31. Skevington SM, Lotfy M, O'Connell KA. The World Health Organization's WHOQOL-BREF quality of life assessment: psychometric properties and results of the international field trial. A report from the WHOQOL group. Qual Life Res. 2004;13:299-310.

32. Ferrans CE, Powers MJ. Psychometric assessment of the Quality of Life Index. Res Nurs Health. 1992;15:29-38.

33. Grosse SD, Wordsworth S, Payne K. Economic methods for valuing the outcomes of genetic testing: beyond cost-effectiveness analysis. Genet Med. 2008;10:648-54.

34. Rogowski WH, Grosse SD, John J, Kääriäinen H, Kent A, Kristofferson $\mathrm{U}$, et al. Points to consider in assessing and appraising predictive genetic tests. J Community Genet. 2010;1:185-94.

35. Rapley M. Quality of life research. London: SAGE; 2003.

36. Joseph L, Cankovic M, Caughron S, et al. The spectrum of clinical utilities in molecular pathology testing procedures for inherited conditions and cancer: a report of the association for molecular pathology. J Mol Diagn. 2016;18:605-19. 